\title{
Biofertilizantes una alternativa ecológica para la agricultura frente al cambio climático en el Ecuador
}

\section{Biofertilizers an ecological alternative for agriculture in the face of climate change in Ecuador}

\section{Biofertilizantes uma alternativa ecológica para a agricultura contra as mudanças climáticas no Equador}

\author{
Karla F. Solis-Charcopa ${ }^{\text {I }}$ \\ karlafsolis@gmail.com \\ Freddy Quiroz-Ponce II \\ freddyquirozp@gmail.com \\ Lucia M. Vernaza-Quiñonez ${ }^{\text {III }}$ \\ luciavernaza@gmail.com \\ Felipe Carrera-Villacrés IV \\ felipecavillacres@gmail.com
}

Recibido: 30 de enero de 2017 * Corregido: 20 de febrero de 2017 * Aceptado: 20 junio de 2017

\footnotetext{
I Docente Investigadora de la Escuela Gestión Ambiental de la Pontifica Universidad Católica del Ecuador, Sede Esmeraldas, Esmeraldas, Ecuador.

II Docente Investigadora de la Escuela Gestión Ambiental de la Pontifica Universidad Católica del Ecuador, Sede Esmeraldas, Esmeraldas, Ecuador.

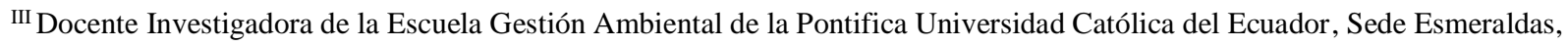
Esmeraldas, Ecuador.

IV Doctorado en Desarrollo y Medio Ambiente de la Universidad Federal do Ceará, Brasil.
} 


\section{Resumen}

Los efectos negativos del cambio climático en la agricultura podrían ser significativos en países en desarrollo como Ecuador. Estos efectos negativos en la agricultura podrían generar dos problemas diferentes en Ecuador. El primero podría ser el impacto negativo del clima en el cultivo, lo que ocasionaría la pérdida de cosecha. El segundo podría ser un impacto negativo en la economía ecuatoriana porque este sector representa la segunda fuente de ingresos más grande para el país. Para abordar este problema mundial y sus posibles impactos en la industria agrícola ecuatoriana, se realizaron pruebas de campo para analizar parámetros como el crecimiento radicular y altura de planta mediante el uso de bio-fertilizantes en el cultivo de frejol caupí (Vigna unguiculata L.). El objetivo es dar a conocer alternativas sustentables y sostenibles para el ambiente con la finalidad de reducir el uso de fertilizantes y químicos en la industria agrícola del Ecuador logrando así reducir los impactos del cambio climático.

Palabras clave: Cambio climático; agricultura; bio-fertilizante; micorrizas. 


\section{Abstract}

The negative effects of climate change on agriculture could be significant in developing countries such as Ecuador. These negative effects on agriculture could generate two different problems in Ecuador. The first could be the negative impact of climate on the crop, which would lead to crop loss. The second could be a negative impact on the Ecuadorian economy because this sector represents the second largest source of income for the country. In order to address this global problem and its possible impacts on the Ecuadorian agricultural industry, field tests were performed to analyze parameters such as root growth and plant height through the use of biofertilizers in cowpea (Vigna unguiculata L.) . The objective is to present sustainable and sustainable alternatives for the environment with the aim of reducing the use of fertilizers and chemicals in Ecuador's agricultural industry, thus reducing the impacts of climate change.

Key words: Climate change; farming; biofertilizer; mycorrhizae. 


\section{Introducción.}

El cambio climático se ha convertido en un gran problema público, donde todos hablan del calentamiento global y de cómo medir sus impactos. ¿Pero qué significará el cambio climático en diferentes partes del mundo y sectores específicos como la agricultura? En los países en desarrollo, algunos agricultores se enfrentan a un futuro incierto porque no tienen información sobre los diferentes escenarios negativos o positivos que el cambio climático podría traer en el largo plazo (Morton, 2007). Por esta razón, este trabajo se enfoca en analizar los efectos del uso de biofertilizantes en la producción agrícola en los países en desarrollo mediante la aplicación de micorrizas en el cultivo de frejol caupi (Vigna unguiculata L.), para disminuir el uso de fertilizantes y químicos que generan impactos negativos en el ambiente; evidenciando alternativas viables para mitigar el cambio climático en la producción agrícola e impactos negativos en la economía del Ecuador .

Una población mundial en constante crecimiento y el cambio climático son dos de los problemas más importantes que conllevan a otros problemas como la pobreza, la inseguridad alimentaria y la inestabilidad económica (Jones, Hansen, Royce, y Messina, 2000). El sector agrícola representa la principal fuente de apoyo para la seguridad alimentaria y la economía de los países en desarrollo (Stringer y Pingali, 2004). Sin embargo, las actividades de este sector, como la extracción de recursos naturales o las actividades agrícolas, están aumentando la emisión de dióxido de carbono $\left(\mathrm{CO}_{2}\right)$, metano $\left(\mathrm{CH}_{4}\right)$, óxido nitroso $\left(\mathrm{N}_{2} \mathrm{O}\right)$ y los halocarburos (gases que contienen flúor, cloro y bromo ); estos gases se acumulan en la atmósfera, causando el cambio climático (Palanichamy, 2011; Pandey, 2009). Como resultado, el mundo se enfrenta al calentamiento global y los consiguientes impactos negativos, como cambios en la temperatura o patrones inusuales de 
precipitación (Solomon, 2007). Todos estos cambios podrían afectar, la producción agrícola ecuatoriana de manera negativa o positiva. Por ejemplo, los eventos climáticos ya se han producido en las Pampas argentinas entre 2000 y 2002, o la sequía de la Amazonia en 2005 (Parry, 2007). Sin embargo, estos impactos podrían medirse considerando la ubicación geográfica de cada país (Cline, 2007). Además, somos testigos de los efectos del cambio climático, como lo es la pérdida de biodiversidad (Smith et al., 2007), sequías (Ogallo, Boulahya, y Keane, 2000), disminución de los servicios ecosistemicos (Nelson et al., 2013), el aumento del nivel del mar y la disminución del rendimiento de los cultivos (Hoffmann, 2011) y los incrementos de la temperatura y los descensos de la precipitación (Calzadilla et al., 2013), que podrían empeorar el hambre y la seguridad alimentaria en el mundo (Cline, 2007). De hecho, los parámetros agrícolas, como el rendimiento de los cultivos, están disminuyendo en todo el mundo. Además, como resultado de estos escenarios, algunos productos agrícolas podrían recibir un incremento en el precio (Rosenzweig y Parry, 1994). Elbehri, Genest, y Burfisher (2011) han expresado su preocupación, ya que ilustran las diferentes presiones que los efectos del cambio climático están causando para los cultivos. Los países en desarrollo podrían verse gravemente afectados por el cambio climático. El motivo no se debe a la ubicación geográfica, sino a las características económicas que estos países deben enfrentar para este problema global (Berg, de Noblet-Ducoudré, Sultan, Lengaigne, y Guimberteau, 2013), especialmente porque la agricultura ha sido la principal fuerza económica de muchos países en desarrollo (Toulmin, International African, Royal African, y Social Science Research, 2009). El sector agrícola en el Ecuador representa el 4\% del Producto Interno Bruto Global (PIB) en todo el mundo (World Bank, 2014). Por esta razón, se considera como una fuente principal de ingresos (Johnston and Mellor, citado en Stringer, 2001). En Ecuador, la agricultura sigue teniendo un papel importante en la economía, ya que representa el segundo mayor sector de exportaciones, que 
proporciona el 6,9\% del PIB y representa casi el 10\% de las personas ecuatorianas económicamente activas (World Bank, 2008). Sin embargo, antes de la década de 1970, la agricultura era la principal fuente de exportación e ingreso para el gobierno ecuatoriano (Ludena \& Wong, 2006). A pesar de eso, como país en desarrollo, Ecuador requirió información específica para tomar medidas relacionadas con el tema del calentamiento global, donde las modulaciones sobre la producción agrícola podrían describir con precisión el cambio climático y su efecto en la agricultura. Sin embargo, los países en desarrollo no tienen recursos suficientes para adquirir equipos para monitorear las variables climáticas, lo que dificulta la implementación de estrategias (Mukheibir, 2007).

\section{Efectos del cambio climático en la producción agrícola}

Cada vez hay más pruebas de que el cambio climático podría afectar a la industria agrícola. Por ejemplo, la producción de cultivos podría verse afectada por las variables climatológicas, como el aumento de las temperaturas, la precipitación variable y el aumento de los niveles de dióxido de carbono en la atmósfera (Parry, Rosenzweig, Iglesias, Livermore, y Fischer, 2004).Además, algunas proyecciones explican que el aumento de la temperatura hará que disminuya la cantidad de biomasa absorbida por el cultivo (Berg et al., 2013, p. 94). Además, la precipitación va a disminuir, en algunos casos provocando sequías, que causarán problemas a los cultivos por la falta de agua. Esta información es consistente con las ideas de Hoffmann (2011, p. 3) el cual indica que los efectos del cambio climático en la producción agrícola se presentarán de la siguiente manera: aumento de plagas, alta probabilidad de aridez y degradación de la tierra, cambios en la producción agrícola y ganadera, y escasez de agua. Todos estos factores, como consecuencia del aumento de la temperatura, el aumento del nivel de $\mathrm{CO} 2$, los cambios en los patrones de precipitación y el aumento 
del nivel del mar, son factores negativos para la producción agrícola de los países en desarrollo. Sin embargo, dentro de estos escenarios negativos del cambio climático en la agricultura, el aumento de la temperatura podría ser beneficioso para la fotosíntesis de las plantas (Berg, Sultan, y NobletDucoudré, 2010). En consecuencia, los efectos negativos del cambio climático llevan a otros efectos negativos en el sector agrícola. Por ejemplo, en Mozambique entre 1999 y 2000, el crecimiento económico disminuyó del ocho por ciento, debido a que algunos cultivos sufrieron graves daños como resultado de las inundaciones en 2000 (Comission for Africa, 2005).Otros modelos sugieren que la disminución de la producción de alimentos provocará un aumento en los precios de los productos básicos como el azúcar, el arroz y el maíz (Rosenzweig y Parry, 1994, p. 137). Como resultado, los índices económicos de algunos países en desarrollo podrían sufrir variaciones cada año, dependiendo del clima proyectado, que afecte el bienestar de su población. Esta posición fue apoyada por Calzadilla et al. (2013), quien señaló información similar en otros países en desarrollo.

\section{Adaptación y mitigación del cambio climático en Ecuador}

El mundo es más consciente de los impactos del cambio climático (Watson, citado en Mendelsohn y Dinar, 1999). Sin embargo, la capacidad de adaptación y vulnerabilidad de cada país definirá su experiencia con respecto a estos impactos (Mertz, Halsnaes, Olesen, y Rasmussen, 2009). Por esta razón, el significado y la comprensión de la adaptación y la mitigación juegan un papel principal en la identificación de soluciones para este problema (Barker, 2003). En Ecuador, para reducir los impactos del cambio climático se han sugerido muchas alternativas como la zonificación agroecológica, las temporadas de cosecha y la instalación del sistema de riego (NCEcuador, citado en Parry, 2007). Sin embargo, antes de hacer cualquier tipo de inversión en tecnologías como el sistema de riego en Ecuador, es necesario saber si estos impactos del cambio 
climático van a tener impactos negativos y significativos en la economía y el sector agrícola de Ecuador. A pesar de eso, Ecuador ya ha creado diferentes programas para mitigar el cambio climático como la promoción de sistemas silvopastorales, la agroforestería, la gestión de ecosistemas frágiles, la mejora de los animales y el manejo del estiércol en biodigestores en el área forestal y ganadera (Viteri y Cordero, 2012)

\section{Direccionando alternativas ecológicas}

Muchos estudios indican que el cambio climático tendrá efectos negativos y positivos en personas de todo el mundo (Calzadilla et al., 2013). Mientras, otros estudios explican que los países en desarrollo son los más vulnerables a estos cambios debido a sus características económicas (Berg et al., 2013). Por ejemplo, algunos países como Ecuador ya están tomando algunas iniciativas y políticas para abordar los efectos del cambio climático en el sector agrícola (Ministerio del Ambiente del Ecuador, 2012). Sin embargo, es necesario tomar estas decisiones con estudios investigativos de campo como lo es las pruebas evidenciadas sobre el uso de micorrizas como alternativa ambiental frente a la necesidad de reducir fertilizantes y químicos. El objetivo de este estudio es tratar de abordar y llenar esta importante brecha. A través de la exploración de estudios de campos que presenten alternativas sólidas a los pequeños agricultores que son los más vulnerables a este problema global. Promoviendo interrogantes como:

1. ¿Qué sabemos sobre los efectos del cambio climático en el sector agrícola de Ecuador?

2. ¿El sector agrícola en el Ecuador tiene evidenciado alternativas de productos para mantener rendimientos y productividad frente a la variabilidad climática de la precipitación y la temperatura? 
3. ¿Cuál será el impacto a escala económica del cambio climático en la economía ecuatoriana, considerando que el sector agrícola es la segunda fuente de ingresos más grande para el gobierno ecuatoriano?

\section{Discusión y Conclusión}

¿Qué significa el cambio climático para diferentes partes del mundo y sectores específicos como la agricultura? La literatura analizada indica que los sectores agrícolas en los países en desarrollo serán susceptibles a los efectos del cambio climático. Además, en estos países, los agricultores enfrentan un futuro incierto porque no tienen información sobre los diferentes escenarios negativos o positivos que el cambio climático podría traer a largo plazo (Morton, 2007). Desde esta perspectiva, el sector agrícola es muy importante para la economía y el bienestar del pueblo ecuatoriano. El objetivo de esta investigación fue contribuir con datos de campos relacionados al uso de micorrizas en cultivos como el frejol caupi (Vigna unguiculata L.) como medida paliativa para mitigar los impactos ambientales, por el uso intensivo de fertilizantes y químicos en la agricultura. Para ello se realizó la aplicación de dos tipos de micorrizas (Glomus etunicatum, Glomus intraradices, Glomus losciculatum con un 50\% infección en el cultivo (Biofertilizante 1) y, Acaulospora spp., Entrophospora spp., y Gigaspora spp con un $80 \%$ de infección en el cultivo (Biofertilizante 2)), ambos géneros de micorrizas fueron colocados en el cultivo de frejol caupí (Vigna unguiculata L.). Así mismo el comportamiento de estos dos géneros con diferente grados de infección fueron comparados con un testigo, con la finalidad de evaluar parámetros de crecimiento radicular y altura de planta por tratamiento, en un periodo de 94 días durante la época de Marzo a Junio. 
Las mediciones radiculares (Figura 1) en promedio evidencian que las plantas infectadas con los géneros de micorrizas presentan un mayor crecimiento radicular que las presentadas por las plantas utilizadas en el tratamiento testigo en un periodo de valuación de 94 días. Este crecimiento radicular coincide con estudios realizados por Marro, Lax, Cabello, Doucet \& Becerra (2014) los cuales indican que existe un mayor crecimiento radicular en cultivos agrícolas. Estos resultados incentivan a una disminución del uso de fertilizantes y químicos, debido a que los resultados son favorables para con las características físicas de las plantas, permitiéndole así estar más preparada para enfrentar diversas situaciones de campo.

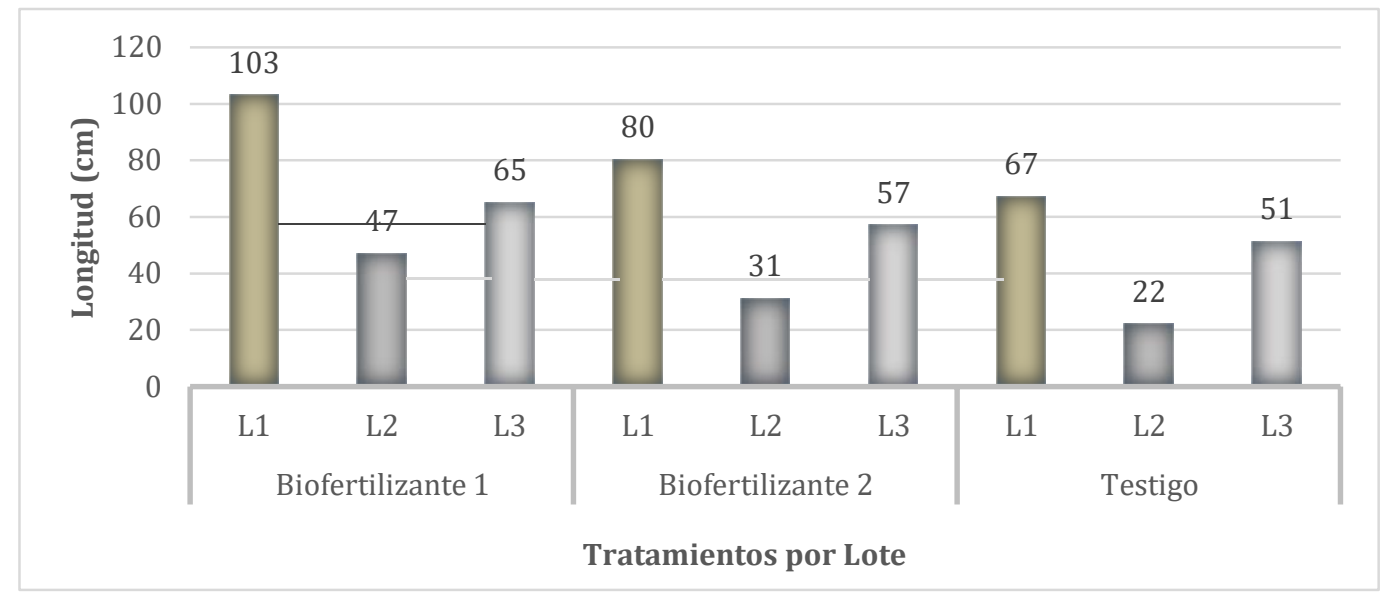

Figura 1. Crecimiento Radicular por Tratamientos

Mediante la aplicación de micorrizas la evaluación de altura de planta es favorable si se compara con el testigo (Tabla 1). Los resultados a firman lo expuesto por Barrer (2009) y Montes, Solis \& Quintos (2011), quienes indica que las micorrizas promueven la tasa de crecimiento de la planta, lo cual es favorable por que incentiva a un mejor desenvolvimiento general del cultivo, desencadenando una mayor productividad agrícola. 
Tabla 1.- Altura promedio de plantas trasplantadas

\begin{tabular}{|c|c|c|c|c|c|c|c|c|c|}
\hline & & \multicolumn{8}{|c|}{ Evaluación (días/cm) } \\
\hline Tratamientos & Lotes & D17 & D24 & D31 & D38 & D45 & D52 & D59 & D66 \\
\hline \multirow{3}{*}{$\begin{array}{c}\text { Biofertilizante } \\
1\end{array}$} & L1 & 21 & 29 & 39 & 50 & 60 & 69 & 87 & 102 \\
\hline & L2 & 19 & 22 & 27 & 33 & 37 & 38 & 39 & 42 \\
\hline & L3 & 20 & 27 & 34 & 43 & 49 & 55 & 63 & 71 \\
\hline \multirow{3}{*}{$\begin{array}{c}\text { Bioferilizante } \\
2\end{array}$} & L1 & 18 & 25 & 32 & 47 & $\underline{54}$ & 61 & 80 & 91 \\
\hline & $\mathrm{L} 2$ & 19 & 20 & 25 & 29 & 34 & 38 & 40 & 40 \\
\hline & L3 & 19 & 25 & 30 & 37 & 43 & 50 & 59 & 64 \\
\hline \multirow[t]{3}{*}{ Testigo } & L1 & 18 & 25 & 30 & 43 & 50 & 60 & 66 & 90 \\
\hline & L2 & 16 & 19 & 23 & 28 & 33 & 34 & 38 & 40 \\
\hline & L3 & 16 & 23 & 27 & 33 & 44 & 48 & 55 & 62 \\
\hline
\end{tabular}

Este documento ha expuesto algunas importantes evidencias en cuanto a parámetros agrícolas incentivando el uso de bio-fertilizantes como alternativa ecológica para mitigar los efectos del cambio climático en la producción agrícola en el Ecuador, mediante el incentivo al uso de micorrizas como alternativa para disminuir el uso de fertilizantes y químicos. Se expuso que los efectos sobre la agricultura son el aumento de las plagas, la alta probabilidad de aridez y degradación de la tierra, los cambios en los cultivos y la producción ganadera y la escasez de agua, que afectan la producción agrícola (Hoffmann, 2011). Como consecuencia del aumento de las temperaturas, aumento del nivel de $\mathrm{CO} 2$, cambios en los patrones de precipitación y cambios en el aumento del nivel del mar. Por lo tanto, el gobierno ecuatoriano requiere muchas acciones como mitigación y adaptación al cambio climático. Sin embargo, antes de hacer esos pasos, es muy importante tener una idea de en qué medida y los tipos de cambios en el sector agrícola se verán afectados por el cambio climático. Para afrontar con argumentos este tema global, en condiciones climáticas reales de Ecuador. Teniendo en cuenta que el gobierno actual tiene un alto nivel de inversión en el sector agrícola (MAGAP, 2013), y que Ecuador podría mejorar sus índices de economía, dando un fuerte apoyo a los pequeños agricultores, que son los grupos más vulnerables a enfrentar con los impactos negativos del cambio climático. 


\section{Bibliografía.}

Barker, T. (2003). Representing global climate change, adaptation and mitigation. Global Environmental Change, 13(1), 1-6. doi: 10.1016/S0959-3780(02)00085-7

Berg, A., de Noblet-Ducoudré, N., Sultan, B., Lengaigne, M., \& Guimberteau, M. (2013). Projections of climate change impacts on potential $\mathrm{C} 4$ crop productivity over tropical regions. Agricultural and Forest Meteorology, 170, 89-102. doi: 10.1016/j.agrformet.2011.12.003

Berg, A., Sultan, B., \& Noblet-Ducoudré, N. (2010). Including tropical croplands in a terrestrial biosphere model: application to West Africa. Climatic change, 104(3-4), 755-782. doi: 10.1007/s10584-0109874-x

Calzadilla, A., Rehdanz, K., Betts, R., Falloon, P., Wiltshire, A., \& Tol, R. S. (2013). Climate change impacts on global agriculture. Climatic change, 120(1-2), 357-374. doi: 10.1007/s10584-013-0822-4

Cline, W. R. (2007). Global warming and agriculture: Impact estimates by country: Peterson Institute.

Comission for Africa. (2005). Our Common Interest: Report of the Commission for Africa. London: Commission for Africa.

Elbehri, A., Genest, A., \& Burfisher, M. E. (2011). Global action on climate change in agriculture: Linkages to food security, markets and trade policies in developing countries: Trade and Markets Division, Food and Agriculture Organization of the United Nations.

Hoffmann, U. (2011). Assuring food security in developing countries under the challenges of climate change: Key trade and development issues of a fundamental transformation of agriculture.

Jones, J. W., Hansen, J. W., Royce, F. S., \& Messina, C. D. (2000). Potential benefits of climate forecasting to agriculture. Agriculture, Ecosystems \& Environment, 82(1), 169-184. doi: 10.1016/S01678809(00)00225-5

Ludena, C., \& Wong, S. (2006). Domestic support policies for agriculture in Ecuador and the US-Andean countries free trade agreement: an applied general equilibrium assessment. Paper presented at the American Agricultural Economics Association 2006 Annual meeting, Selected Paper, Long Beach, CA.

MAGAP. (2013). MAGAP presentó programa de inversion forestal, en cumbre internacional de medio ambiente. from http://www.agricultura.gob.ec

Marro, N., Lax, P., Cabello, M., Doucet, M., \& Becerra, A. (2014). Use of the arbuscular mycorrhizal fungus Glomus intraradices as biological control agent of the nematode Nacobbus aberrans parasitizing tomato. Scielo, 57(5). Obtenido de http://www.scielo.br/scielo.php?script=sci_arttext\&pid=S1516-89132014000500668.

Mertz, O., Halsnaes, K., Olesen, J. E., \& Rasmussen, K. (2009). Adaptation to climate change in developing countries. Environ Manage, 43(5), 743-752. doi: 10.1007/s00267-008-9259-3

Morton, J. F. (2007). The impact of climate change on smallholder and subsistence agriculture. Proceedings of the National Academy of Sciences, 104(50), 19680-19685. doi: 10.1073/pnas.0701855104 
Mukheibir, P. (2007). Qualitative assessment of municipal water resource management strategies under climate impacts: The case of the Northern Cape, South Africa: short communication. Water Sa, 33(4), 575-581.

Nambiza, W. (2013). Baseline study for the Climate Change, Agriculture and Poverty Alleviation (CCAP) initiative. from http://www.tfcg.org/pdf/TFCG CCAP Baseline Survey Report 2013 FINAL.pdf

Nelson, E., Kareiva, P., Ruckelshaus, M., Arkema, K., Geller, G., Girvetz, E., . . Tallis, H. (2013). Climate change's impact on key ecosystem services and the human well-being they support in the US. Ecol Environ, 11(9), 483-493. doi: 10.1890/120312

Ogallo, L. A., Boulahya, M. S., \& Keane, T. (2000). Applications of seasonal to interannual climate prediction in agricultural planning and operations. Agricultural and Forest Meteorology, 103(1), 159166. doi: 10.1016/S0168-1923(00)00109-X

Palanichamy, A. (2011). Global warming and impact on Indian agriculture and food production. Food Biology, 1(1).

Pandey, S. (2009). Adaptation to and mitigation of climate change in agriculture in developing countries. IOPscience, 8(1), 012020. doi: 10.1088/1755-1315/8/1/012020

Parry, M. L. (2007). Climate Change 2007: impacts, adaptation and vulnerability: contribution of Working Group II to the fourth assessment report of the Intergovernmental Panel on Climate Change (Vol. 4): Cambridge University Press.

Parry, M. L., Rosenzweig, C., Iglesias, A., Livermore, M., \& Fischer, G. (2004). Effects of climate change on global food production under SRES emissions and socio-economic scenarios. Global Environmental Change, 14(1), 53-67. doi: 10.1016/j.gloenvcha.2003.10.008

Rosenzweig, C., \& Parry, M. L. (1994). Potential impact of climate change on world food supply. Nature, 367(6459), 133-138. doi: 10.1038/367133a0

Smith, P., Martino, D., Cai, Z., Gwary, D., Janzen, H., Kumar, P., . . O, S. (2007). Climate Change $2007-$ Mitigation of Climate Change: Working Group III Contribution to the Fourth Assessment Report of the IPCC: Cambridge University Press.

Solomon, S. (2007). Climate change 2007-the physical science basis: Working group I contribution to the fourth assessment report of the IPCC (Vol. 4): Cambridge University Press.

Stringer, R. (2001). How Important are the Non-traditional Economic Roles of Agriculture in Development? : Centre for International Economic Studies.

Stringer, R., \& Pingali, P. (2004). Agriculture's Contributions to Economic and Social Development.

Toulmin, C., International African, I., Royal African, S., \& Social Science Research, C. (2009). Climate change in Africa. New York; London: Zed Books in association with International African Institute, Royal African Society, Social Science Research Council.

Viteri, A., \& Cordero, E. V. (2012). Documento de Análisis del Sector Forestal en el Contexto de Adaptación y Mitigación al Cambio Climatico del Sector Uso del Suelo, Cambio de Suelo, y Silvicultura (Forestal) en el Ecuador. Ministerio del Ambiente, República de Ecuador, Cordero, V., \& Cuenca, P., 6, 5-7. 
Biofertilizantes una alternativa ecológica para la agricultura frente al cambio climático en el Ecuador

World Bank. (2008). World Development Report 2008: Focus F: Adaptation to and mitigation of climate change in agriculture. from http://go.worldbank.org/C3TCZPDAJ0

World Bank. (2014). Ecuador dashboard: impacts \& vulnerabilities. from http://sdwebx.worldbank.org/climateportalb/home.cfm?page=country_profile\&CCode=ECU\&ThisTa $\underline{\mathrm{b}=\text { ImpactsVulnerabilities }}$ 